\title{
Wireless control of induced radiofrequency currents in active implantable medical devices during MRI
}

\author{
Volkan Acikel $^{1}$ (D) | Berk Silemek $^{2}$ (D) | Ergin Atalar ${ }^{2,3}$ \\ ${ }^{1}$ Aselsan, REHIS, Ankara, Turkey \\ ${ }^{2}$ National Magnetic Resonance Research Center (UMRAM), Bilkent University, Ankara, Turkey \\ ${ }^{3}$ Department of Electrical and Electronics Engineering, Bilkent University, Ankara, Turkey
}

\section{Correspondence}

Volkan Acikel, Konya Yolu 8. Km, Oğulbey Mah. 3051. Sok. No.3, 06830

Ankara, Türkiye.

Email: volkan.acikel@gmail.com
Purpose: To introduce a prototype active implantable medical device (AIMD) for which the induced radiofrequency currents can be controlled wirelessly.

Methods: The modified transmission line method is used to formulate how the lead-case impedance of an AIMD affects the temperature rise around the electrode. A prototype AIMD is designed with the aim of controlling the unwanted temperature rise around its electrode during an MRI examination by altering the impedance between the lead and the case of the implant. MRI experiments were conducted with this prototype implant, which also has a built-in temperature sensor at its electrode. During the experiment, the implant's lead-case impedance was controlled using Bluetooth communication with a remote computer, and the lead tip temperature was recorded.

Results: Ten different lead-case impedance values and their corresponding tip temperature rises were examined during MRI experiments. The experimental results confirmed that the tip temperature rise can be controlled by varying the lead-case impedance wirelessly.

Conclusion: The feedback from the temperature at the AIMD tip, together with variable lead-case impedance, enables control of the safety profile of the AIMD during an MRI examination.

\section{K E Y W O R D S}

active implantable medical devices, Bluetooth, heating reduction, MoTLiM, MR heating, RF safety

\section{1 | INTRODUCTION}

Radiofrequency (RF)-induced excessive tissue heating is a significant safety problem for patients with active implantable medical devices (AIMDs) who undergo MRI examination. There are 2 case reports about injuries of patients with deep brain stimulator (DBS) implants during MRI examination. ${ }^{1,2}$ Although the RF safety problem of MRI of patients with implants is very well known, the desire to image such patients is overwhelming because of the high diagnostic capability of MRI.
The MRI compatibility of AIMDs, such as pacemakers, DBSs, and spinal cord stimulators, has been under investigation for over 2 decades. ${ }^{3}$ It is well known that RF-induced tissue heating is caused by coupling of the long conductors of the AIMD with the RF coil of the MRI scanner. This is often called the "antenna effect." 4 The properties of the RF transmit coil, such as the magnitude and phase distribution of the electric field (E-field), affect the amount of RF heating. ${ }^{5}$ In addition, the AIMD lead properties, such as the insulation thickness, length of the wire, and dielectric constant of the insulator, affect the amount of RF-induced heating 
significantly. ${ }^{6}$ Another effect can be considered as a lead pathway through the body. ${ }^{6}$ A detailed theoretical analysis of the problem was conducted by calculating the specific absorption rate around the electrode and the rise in temperature using the bioheat equation. ${ }^{7}$ Moreover, theoretical models in the form of transfer function ${ }^{8-12}$ or distributed circuit element model $^{9,13}$ have been proposed to analyze the problem and predict the temperature rise.

There are 2 main approaches to solve the problem of RF-induced heating. The first is to design leads such that the current induced in them during MRI is insignificant. ${ }^{14-20}$ Some researchers have proposed modifying the geometry of the lead, such as by replacing lead wires with fiber-optic cables. ${ }^{15}$ Another useful approach is the use of resistive leads that have a resistance above $3 \mathrm{k} \Omega$ for approximately $75 \mathrm{~cm}$, enough to keep the rise in temperature below $2{ }^{\circ} \mathrm{C}$ for up to 5 minutes for a $4-\mathrm{W} / \mathrm{kg}$ specific absorption rate (SAR) value. ${ }^{20}$ A possible usage of current traps was demonstrated on catheter leads ${ }^{14}$ and for implant leads. ${ }^{16}$ New enhanced materials are implemented that lowers the temperature by heat-sink effect. ${ }^{18}$ More recently, resistive tapered stripline has been shown. ${ }^{19}$ Using inductance-based ${ }^{17}$ or similar techniques, some commercially available AIMDs have received US Food and Drug Administration approval to undergo MRI scans under some specific conditions. $^{21-23}$

The second approach is to modify the RF transmit field such that the induced currents on the leads are manageable. For example, by using dual-drive birdcage coils,${ }^{24}$ a significant RF-induced heating reduction was demonstrated. A rotating birdcage coil ${ }^{25,26}$ has been proposed to coincide with a null E-field region caused by the linear excitation of these coils around the implant. The linear excitation, however, results in an increased SAR and image distortion; therefore, a solution with multiple transmit channels is being investigated. ${ }^{27-31}$ This growing interest in parallel RF transmit technology brings additional possibilities for safe scans, but also makes the problem more complex.
Considering the severity of previously reported injuries $^{1,2}$ related to AIMDs and the high variability of the conditions that cause high temperature rises, there are limits in the reliability of the above-mentioned methods, which motivates the development of a method of real-time temperature rise control. Because it is not possible to control most of the above-mentioned parameters, such as the lead path, we propose to control the lead-case impedance in real time as we monitor the temperature rise during the scan. In an earlier work, ${ }^{32}$ an AIMD was designed with the capability of recording and sending the temperature data around its electrode in real time with a low-energy wireless channel. In this study, we further modified the AIMD to control the temperature rise by varying the lead-case impedance wirelessly. In the following section, the effect of the impedance between the lead and the case on the rise of the electrode temperature is formulated using the modified transmission line method (MoTLiM). ${ }^{33}$ Later, the design details of the modified AIMD are given. The experimental results show that the RF-induced temperature rise can be altered during an MRI scan without any intervention to the MRI scanner or to the structure of the AIMD.

\section{2 | THEORY}

It is known that the lead-to-case impedance has a significant effect on the induced currents in the implant leads. ${ }^{33,34}$ One of the methods to formulate the relationship between the electrode heating and the lead-case impedance is to use the MoTLiM. ${ }^{13}$ In this model, the lead is assumed to be shielded by the high conductivity of the body, and therefore it can be modeled as if it is a coaxial line. The effect of the electric field along the length of the wire can be modeled as a distributed voltage source along the length of the coaxial cable. The circuit equivalent of the model can be seen in Figure 1 . Using this model, the current in the lead can be calculated as a modified form of the telegrapher equation as (Equation 1):

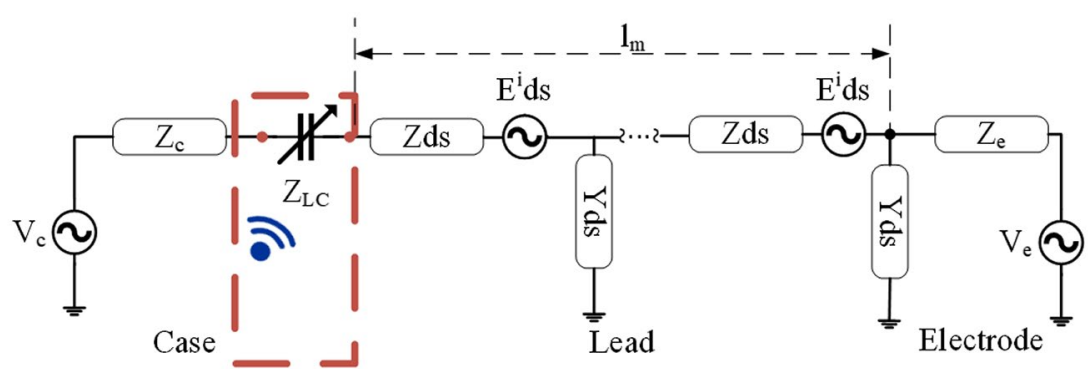

F I G URE 1 The circuit diagram of the MoTLiM model of a variable lead-case impedance. The 3 main components of the MoTLiM model of the AIMD are the case, lead, and electrode, which are shown with lumped circuit elements. The wireless lead-case impedance $Z_{L C}$ alteration was realized with a capacitance network indicated with a variable capacitance symbol within a red-dashed box. The case and electrode were modeled as a voltage source ( $V_{c}$ and $V_{e}$, respectively) and a series impedance $\left(Z_{c}\right.$ and $Z_{e}$, respectively). The characteristic impedance of the lead is $Z$, and the total length is $l_{m} . s$ is the position variable, and $d$ is the derivative operation. $E_{i}$ is the tangential component of the incident electric field 


$$
\mathrm{I}(\mathrm{s})+\frac{1}{\mathrm{k}_{\mathrm{t}}^{2}} \frac{d^{2} I(s)}{d s^{2}}=\frac{E^{i}(s)}{Z}
$$

where $I$ is the current in the lead, $\mathrm{k}_{\mathrm{t}}=\sqrt{-Y Z}$ is the wavenumber of the modified transmission line, $Z$ is the characteristic impedance, and $E^{i}$ is the tangential component of the incident electric field. Additionally, similar to transmission lines, a voltage wave can be defined between the lead and the ground, which is assumed to be at infinity, as follows (Equation 2):

$$
V(s)=\frac{Z}{k_{t}^{2}} \frac{d I(s)}{d(s)}
$$

Although this voltage is hypothetical, it is useful to define boundary conditions for the MoTLiM. In a previous work, ${ }^{33}$ the case and electrode are also modeled using lumped elements (a voltage source and a series impedance), as shown in Figure 1. The previous studies show that under controlled conditions, all parameters in the MoTLiM can be found, and therefore the current in the lead and the electrode can be calculated.

MoTLiM parameters of the lead with insulation were found using the following equations ${ }^{34}$ (Equation 3):

$$
\begin{aligned}
& \mathrm{Z}=k \frac{\eta H_{0}^{(2)}(k \mathrm{~b})-\eta_{d} k_{d} \mathrm{~b} H_{1}^{(2)}(k \mathrm{~b}) \ln (\mathrm{a} / \mathrm{b})}{4} \\
& k_{t}=k \sqrt{\frac{\mathrm{k}_{d}^{2}\left(H_{0}^{(2)}(k \mathrm{~b})-k \mathrm{~b} \ln (\mathrm{a} / \mathrm{b}) H_{1}^{(2)}(k \mathrm{~b})\right)}{\mathrm{k}_{d}^{2} H_{0}^{(2)}(k \mathrm{~b})-k^{3} \mathrm{~b} \ln (\mathrm{a} / \mathrm{b}) H_{1}^{(2)}(k \mathrm{~b})}}
\end{aligned}
$$

where $k$ and $k_{d}$ are the wavenumbers inside the medium and insulation, respectively, $a$ is the radius of the conductor, $b$ is the total radius of the guidewire with insulation, and $\eta$ and $\eta_{d}$ are the wave impedance in the medium and in the insulation, respectively. $H_{i}^{(2)}$ is the $i^{\text {th }}$ order Hankel function of the second kind. The circuit model parameters of the implant case, $Z_{c}$ and $V_{c}$, were found using the method explained in a previous work. ${ }^{33}$ Additionally, the electrode circuit parameters, $Z_{e}$ and $V_{e}$, were found using the same method. More detailed explanations are given in the methods for the analysis of the variable lead-case impedance by using computer simulations.

To calculate the current, Equation 1 must be solved using the following boundary conditions (Equations 4 and 5):

$$
\begin{gathered}
\mathrm{V}_{\mathrm{c}}-\mathrm{Z}_{\mathrm{c}} \mathrm{I}\left(-l_{m} / 2\right)-\mathrm{V}\left(-l_{m} / 2\right)=0 \\
V\left(l_{m} / 2\right)-Z_{e} I\left(l_{m} / 2\right)-V_{e}=0
\end{gathered}
$$

where $Z_{c}$ and $V_{c}$ are the implant case impedance and voltage, respectively, $Z_{e}$ and $V_{e}$ are the electrode impedance and voltage, respectively, and $l_{m}$ is the lead length (see Figure 1). For these boundary conditions, the implant case is assumed to be at location $s=-l_{m} / 2$, and the electrode is assumed to be at location $s=l_{m} / 2$. In this article, after finding all the MoTLiM parameters, the current in the lead is solved and the dissipated power from the real part of the electrode impedance, which will be referred to as dissipated power through the article, is calculated and compared with the temperature rise. Because all the experiments in this article were performed for a perfusionless phantom, the bio-heat equation was not solved. In the formulation, the relationship between dissipated power and the temperature rise is assumed to be linear.

\section{3 | METHODS}

The methods used in this study will be explained in 3 parts. First, the analysis of the effect of the lead-case impedance on the temperature rise at the electrode of the implant will be carried out. Second, the proposed implant design will be explained. Finally, the MR heating experiments for this particular setup will be described.

\section{1 | MoTLiM simulations}

The MoTLiM ${ }^{13}$ was used for the analysis of the effect of altering the connection impedance between lead and case on the rise in the electrode tip temperature. The MoTLiM parameters of the implant, which will be described in the next section, were calculated as described in the Theory section. The method explained in a previous work ${ }^{33}$ was used to extract the circuit model parameters of the implant case and the electrode. A computer-aided design (CAD) model of the case was imported to the electromagnetic (EM) simulation tool, FEKO (FEKO; Altair Engineering Inc., Troy, MI), and was connected to a wire with a diameter of $1 \mathrm{~mm}$. The relative permittivity of the insulation was 3.6. The current at the connection point of the wire and the case was found using EM simulations (FEKO) for 2 wire lengths, 10 and $20 \mathrm{~cm}$. For both simulations, the case and wire were inside a medium with $\varepsilon_{r}=80$ and $\sigma=0.6 \mathrm{~S} / \mathrm{m}$ and were excited with a plane wave that had a unit tangential E-field along the wire. Using these current values and the method defined in a previous work, ${ }^{33}$ the circuit model parameters of the implant case and electrode were calculated. MoTLiM parameters of the lead were calculated for conductor radius $a=0.95 \mathrm{~mm}$ and total radius $b=1.4 \mathrm{~mm}$ using Equation 3. 
After the circuit model parameters of the case, electrode and the MoTLiM parameters of the lead were calculated, the current in the lead was solved for unit, $1 \mathrm{~V} / \mathrm{m}$, tangential electric field incidence, and the dissipated power on the electrode impedance was calculated, then compared with the temperature rise. Because all the experiments in this article were performed for a perfusionless phantom, the bio-heat equation was not solved. In the formulation, the relationship between dissipated power and the temperature rise is assumed to be linear.

The calculations were repeated for each impedance between the case and lead, as shown in Figure 1. The 10 discrete implemented lead-case impedance values were used in these calculations.

To show the effect of the lead-case impedance, the dissipated power was calculated with respect to the lead length. For these calculations, $V_{c}$ was set to $-36.3+j 4.8 \mathrm{mV}$, which was found by the CAD simulations. $Z_{c}$ ranged from $-j 50 \Omega$ to $j 50 \Omega$ in $j 10 \Omega$ steps. All these calculations were carried out under a uniform E-field. The change in the effective electrical length of the lead is demonstrated by the change in the locations of the resonance peaks of the temperature rise. The conductivity of the medium was changed from $0.2 \mathrm{~S} / \mathrm{m}$ to $0.7 \mathrm{~S} / \mathrm{m}$ in $0.1 \mathrm{~S} / \mathrm{m}$ steps, and the relative permittivity was changed from 50 to 90 in steps of 10 . While the other parameters were changed, the case impedance was kept constant at $Z_{\text {case }}=55.26-j 18.88 \Omega$. These calculations were carried out for lead lengths 40 and $90 \mathrm{~cm}$.

\section{2 $\quad$ Temperature sensor implant}

To verify the proposed method, the prototype implant, which has the ability to monitor the temperature of its electrode in real time, ${ }^{32}$ was modified. As in the original design, a titanium alloy case with dimensions of $50 \times 40 \times 10 \mathrm{~mm}$ holds the electronic circuit. The lead length was $40 \mathrm{~cm}$. The temperature sensor was embedded inside the electrode, which is composed of $99.9 \%$ silver with a thickness of $10 \mu \mathrm{m}$ and a $6 \mathrm{~mm}^{2}$ surface area.

A planar inverted-F antenna (PIFA) as a Bluetooth antenna ${ }^{35}$ was added to the circuit of the implant on a $1 \mathrm{~mm}$ thick FR-4 PCB (printed circuit board). A device protection filter was implemented and placed between the PIFA and the Bluetooth chip, a BLE112-A module (Silicon Labs, Austin, TX). The antenna, together with the case and surrounding tissue ( $5 \mathrm{~mm}$ of skin, $10 \mathrm{~mm}$ of fat, and $45 \mathrm{~mm}$ of muscle), was tuned to $2.4 \mathrm{GHz}$ using the CST Microwave Studio (CST; Computer Simulation Technology GmbH, Darmstadt, Germany) electromagnetic simulator. Analysis of the SAR for Bluetooth communication was not carried out because it is known to be very small and is not the focus of this article.

The performance of the PIFA antenna was compared with that of the embedded antenna on the Bluetooth chip.
The device was placed on a table, and the received signal strength indication (RSSI) ${ }^{36}$ was measured for various distances and orientations with its default embedded antenna. Then, the BLE112-A was soldered to the designed PCB, and the Bluetooth antenna was replaced with the PIFA on the PCB. The PCB was placed inside the implant case. The case was closed and sealed to prevent liquid leakage. The implant was placed into a small container $(40 \times 10 \mathrm{~cm})$ filled with salt water $(2 \mathrm{~g} / \mathrm{L})$. The RSSI was measured again at different distances and orientations. For the MR room experiments, the implant was placed as shown in Figure 2A, and the container was filled with a gel phantom (the same phantom used in the RF heating experiments), and the RSSI was measured again. Because the Bluetooth antenna is not the main concern of this study, no further performance analysis was carried out to evaluate it.

To change the lead-case impedance, the shield of the lead was connected to an RF switch network. Three digitally controllable RF switches (PE42442-Z; Peregrine Semiconductor Corporation, San Diego, CA) were used. The RF switches have single-pole 4-throw topology and are arranged as shown in Figure 2B. In total, 10 digital and wireless controllable lead-case impedance values were implemented. Then, the implemented lead-case impedance values were measured for each switch state using a network analyzer (E5061B; Agilent Technologies, Santa Clara, CA). The switch states were changed using custom Bluetooth controller software on the computer. A point-to-point USB optical cable (M2-100; Opticis, Seongnam-si, South Korea) was used for communication to penetrate the Faraday cage.

\section{3 | MRI experiments}

MRI heating experiments were performed using a 1.5T scanner (SM160; Scimedix Inc., Incheon, South Korea). The system's body coil was used as a transmitter. An RF-only sequence (TR, $21 \mathrm{~ms}$; hard RF pulse duration, $6 \mathrm{~ms}$; total time, 484 seconds) was used for the experiments. To compare different experiments within each other, the sequence parameters were kept constant for every configuration, and scanner adjustment was bypassed. The phantom used in the experiments was composed of $20 \mathrm{~g} / \mathrm{L}$ of hydroxyethyl cellulose (HEC), $2 \mathrm{~g} / \mathrm{L}$ of salt; total, $30 \mathrm{~L}$ of HEC gel $(\sigma=0.45 \mathrm{~S} / \mathrm{m}$ and $\varepsilon_{r}=80$ ). After the experimental setup was prepared, the phantom was kept inside the MRI room for 3 hours in order to reach temperature equilibrium before the experiment.

To validate the proposed method, 2 different experimental scenarios were performed. The first scenario was devised to demonstrate the effect of the lead-case impedance on the temperature rise. For this, the RF heating sequence was applied for each lead-case impedance value. The lead-case impedance value was set before the start of the RF heating 

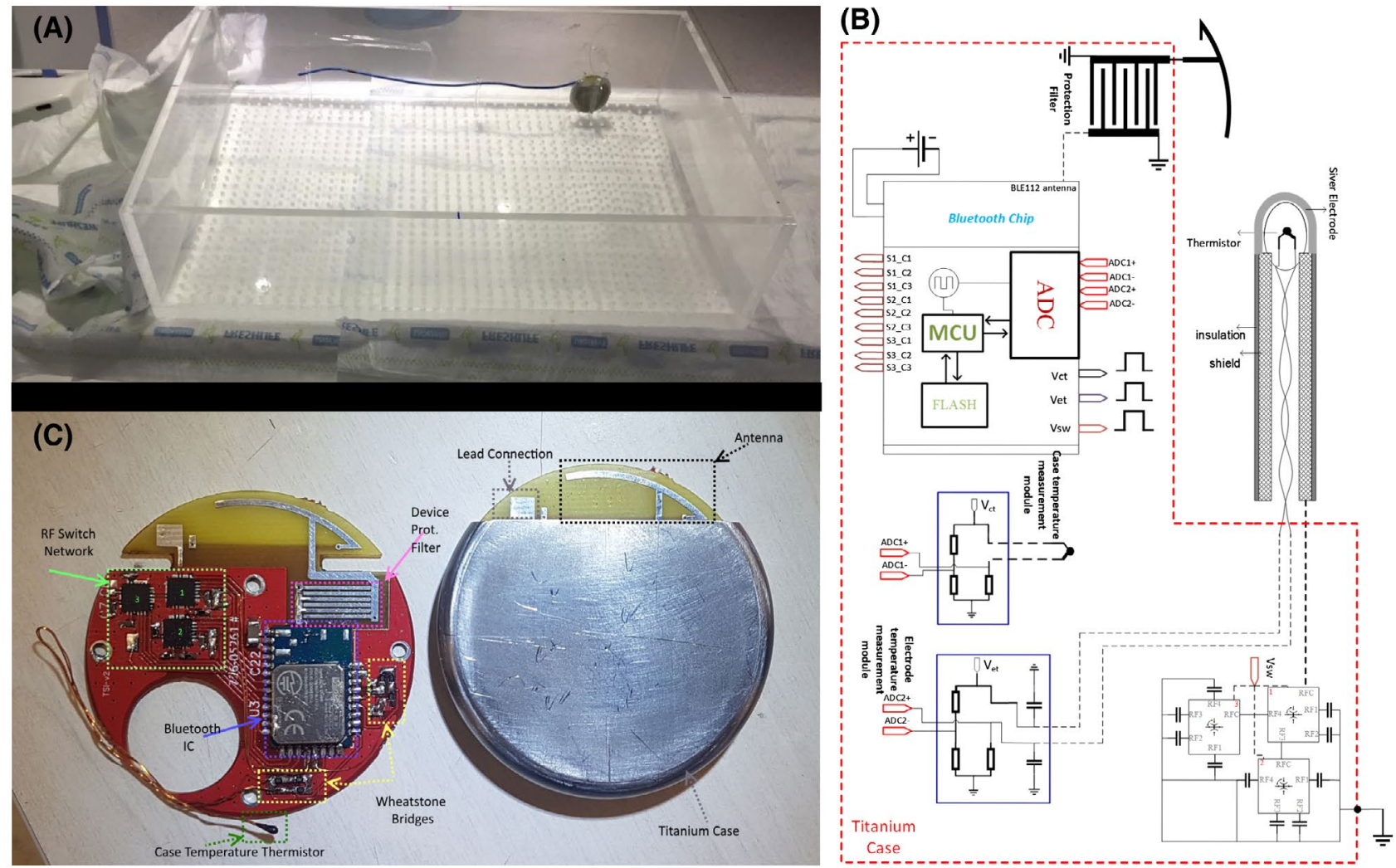

F I G URE 2 (A) Photo of the heating experiment setup without phantom gel. The lead is placed along the z-direction of the magnetic field as far as possible. The implant is close to the edge (30 mm away) of the phantom. In the experiment, the phantom container will be filled almost completely with the phantom material $(\mathrm{h}=145 \mathrm{~mm})$. The center of the phantom was used as a landmark position. (B) Diagram of the modified prototype implant. The RF switches and PIFA are the major modifications. Each RF switch is controlled by 3 digital pins that are connected to the Bluetooth IC. The 3 RF switches are cascaded to implement 10 digitally controllable lead-case impedances. The PIFA antenna is added to the node of the device protection filter. (C) Photo of the implant. Left side: PCB without titanium case. Right side: the same PCB encapsulated with the titanium case. Three RF switches with corresponding switch numbers are outlined in green. Their control pins connected to the Bluetooth chip are outlined in blue. The device protection filter (pink) is connected to the PIFA (black). The lead connection pad near the antenna is indicated with gray. The lead is soldered by this pad, which is connected to the RF switch network. Wheatstone bridges to measure the case and electrode temperatures are shown in yellow. $\mathrm{ADC}=$ analog to digital converter; $\mathrm{MCU}=$ microcontroller unit

sequence. No change in any of the other parameters occurred during the sequence. The temperature at the tip was recorded continuously at $1 \mathrm{~Hz}$. The case temperature was also recorded over 1 period for each lead-case impedance value. After each iteration, enough time (20-30 minutes) was given for the experimental setup to cool down. Then, the next lead-case impedance value was set using wireless commands, and the RF heating sequence was repeated.

The second experiment was devised to demonstrate the wireless controllability of the RF coupling of the AIMD in real time and to illustrate its effect on tip heating. The same experimental parameters were used, except that the total RF sequence time was 18 minutes. Initially, the leadcase impedance value was set to the lowest implemented value, which was set before the start of the sequence. Then, the RF heating sequence started. The temperature rise was monitored on an external computer. Afterward, the leadcase impedance value was set to the highest implemented value. After a few minutes, it was set to its initial lowest value again. Finally, an intermediate lead-case impedance value was set. During the entire procedure, the experimental parameters, especially the applied RF power, were kept the same. Only the lead-case impedance values were adjusted using wireless communication.

\section{4 | RESULTS}

A photo of the implant is shown in Figure 2C, depicting its components. The measured lead-case impedance values for different switch states are given in Table 1. During the experiments, the lead is connected to the implant and sealed. The measurements in the lab with a new antenna have sufficient performance for the MR experiments. The implant residing in the lab test phantom has a maximum of $3 \mathrm{~m}$ of communication distance for the Bluetooth signals. During the 
TA B L E 1 The measured impedance values of the switches at $64 \mathrm{MHz}$

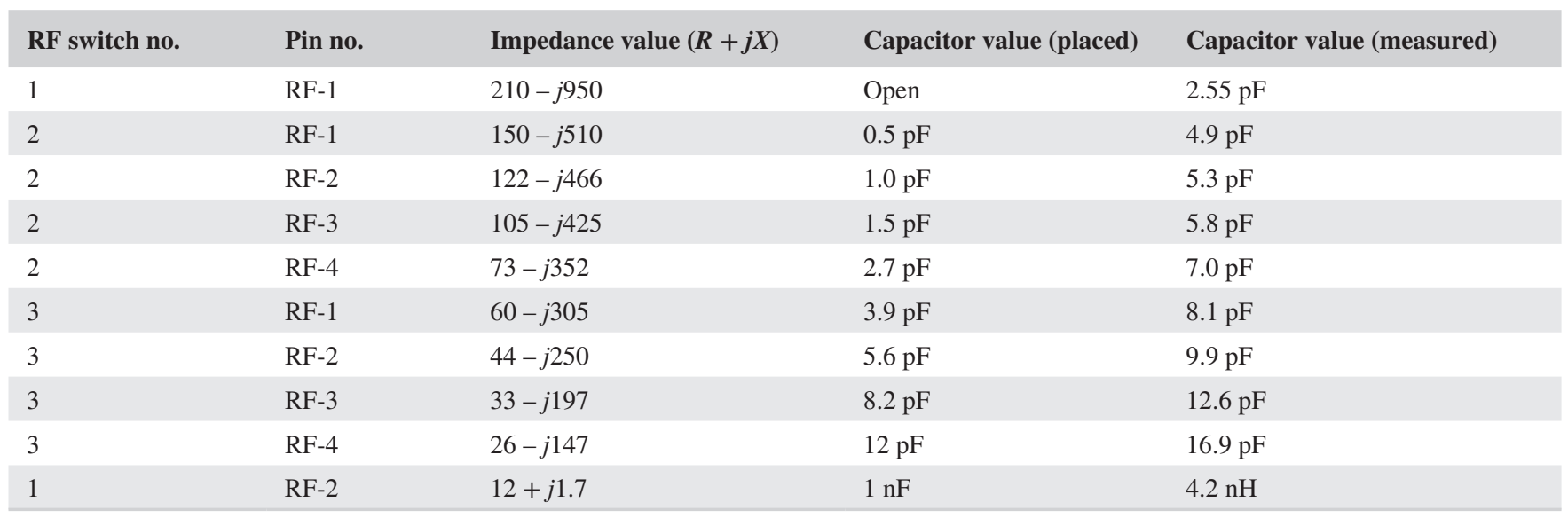

Note: that the measured impedance values include the parasitic impedances of the switches.

TA B L E 2 The baseline tip temperature values, case temperature values, and tip temperature change for each lead-case impedance are given

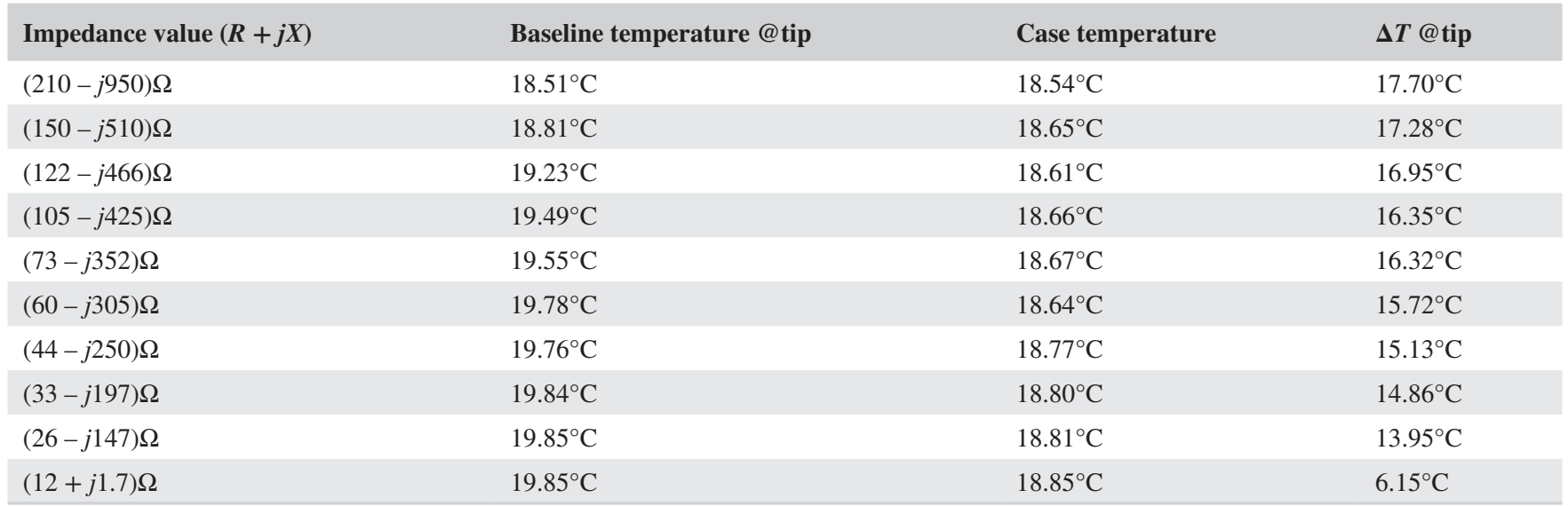

Each iteration consists of 8 minutes of an RF sequence plus 20 to 30 minutes of cool down, which is determined by tip temperature variations of $<0.1^{\circ} \mathrm{C}$.

MR experiments, the maximum communication distance was $2 \mathrm{~m}$. Therefore, the Bluetooth dongle was placed on the edge of the patient table.

After the temperature reached equilibrium at the start of the experiment, the baseline temperature at the tip was $18.5^{\circ} \mathrm{C}$. The value slightly increased for each repetition, reaching $19.8^{\circ} \mathrm{C}$ at the end of the experiment. The case temperature was $18.5^{\circ} \mathrm{C}$ at the start of the experiment and had risen to $18.8^{\circ} \mathrm{C}$ in the last experiment. The corresponding reference temperatures for each lead-case impedance value can be found in Table 2 .

The tip temperature rises measured during the MR heating experiments are given in Figure 3. For this particular experimental setup, the maximum temperature rise was measured when the lead-case impedance value was the highest. The lowest temperature rise was measured when the lead-case impedance value was the lowest. The peak temperature rise decreased by $65 \%$ between these 2 extremes. The temperature rises around the electrode tip at the end of the 8-minute scans are given in Table 2.
The model parameters for the case were calculated as follows: $V_{c}=-36.3+j 4.8 \mathrm{mV}$ for an incident E-field of $1 \mathrm{~V} / \mathrm{m}$ and $Z_{c}=55.26-j 18.88 \Omega$. The electrode parameters were calculated as follows: $V_{e}=-41.3+j 12 \mathrm{mV}$ for an incident E-field of $1 \mathrm{~V} / \mathrm{m}$ and $Z_{e}=82.7-j 3.52 \Omega$. The MoTLiM parameters of the lead were calculated as follows: $k_{t}=7.88$ $j 1.98 \mathrm{~m}^{-1}, Z=80.64+j 334.21 \Omega$.

Using these parameters and the measured lead-case impedances in Table 1, the dissipated power was calculated under a uniform E-field distribution. In Figure 4, the change of the tip temperature rise with respect to lead length is shown for the lead-case impedance values given in Table 1. The arrow indicates the change of the temperature rise for a lead with length $40 \mathrm{~cm}$. In Figure 5, the calculated dissipated power with respect to the lead-case impedance is plotted. Figure 5 also plots the measured tip temperature rise for the same values of the lead-case impedance. Note that although the incident tangential E-field during the experiments are not perfectly uniform along the implant, ${ }^{37}$ they are still useful in predicting the behavior of the temperature. 


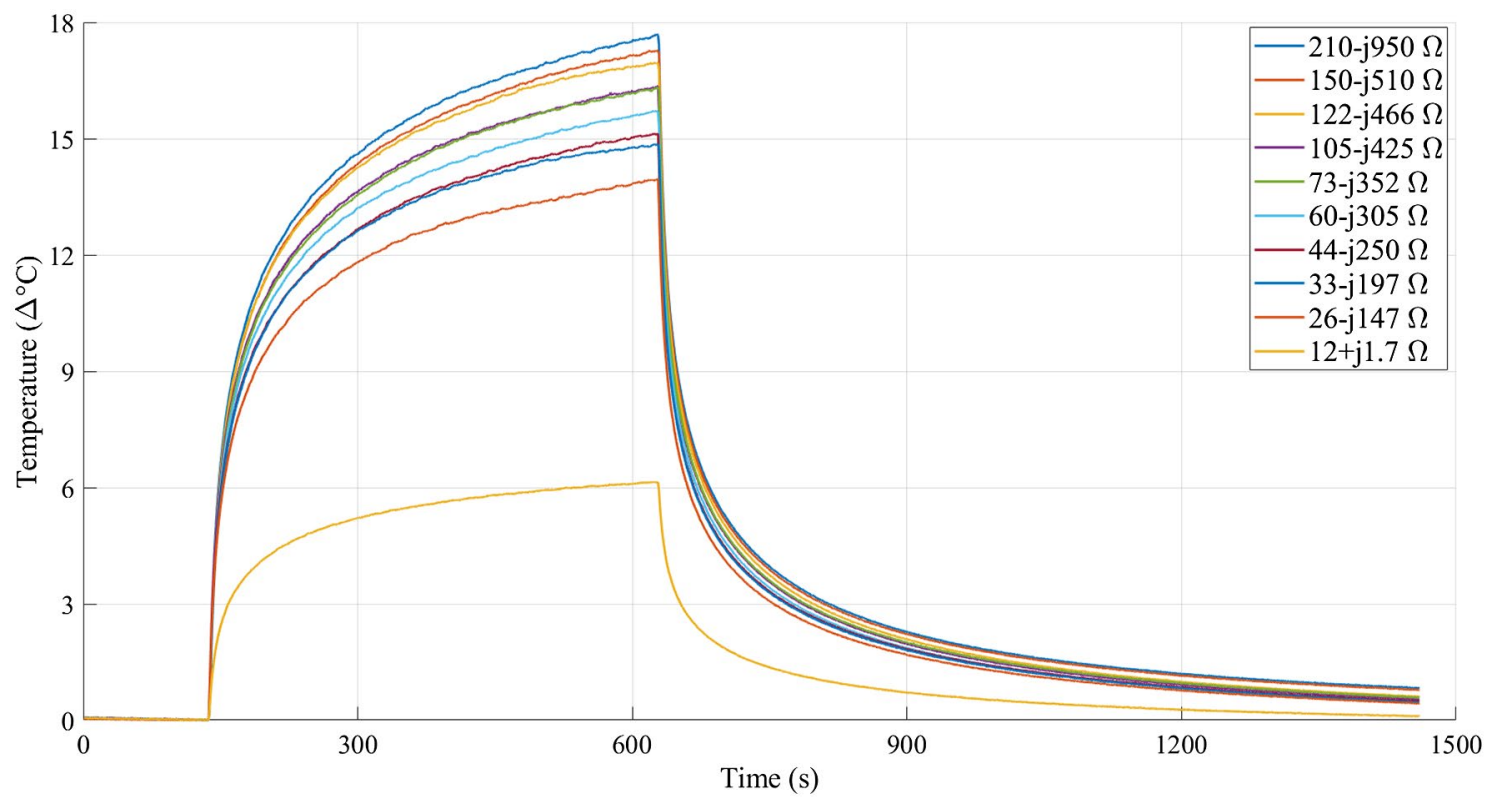

F I G U RE 3 MR heating experiment results for the implemented lead-case impedance values. Ten lead-case impedance values and their corresponding temperature rises are shown. Each lead-case impedance value resulted in a different temperature rise at the tip. For this particular experiment, slight differences in impedance values (at high impedances) change the temperature slightly. When the lead-case impedance was at its lowest, the resulting temperature rise was minimal

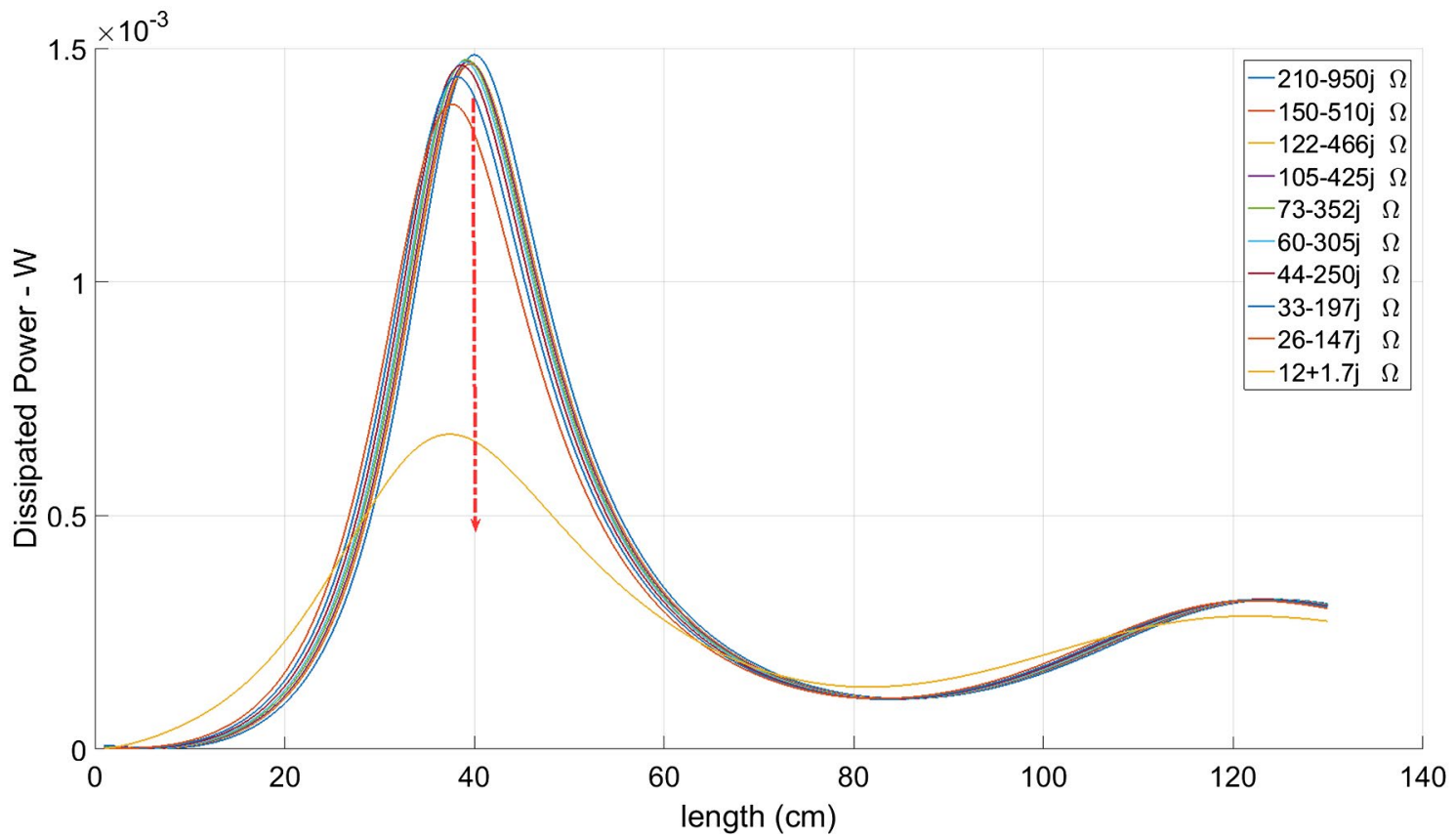

F I G U RE 4 The corresponding dissipated power from the real part of the electrode impedance is plotted as the lead length changes. The power-length curve changes for each lead-case impedance value implemented. The change at $40 \mathrm{~cm}$, which is the implemented implant lead, is illustrated with the arrow

The impedance variation was also analyzed to illustrate the effect of the method. For each lead-case impedance value, the calculated dissipated power value is plotted for each lead length (Figure 4). The resonance length starts to shift, and its peak value decreases when the lead-case impedance decreases. Figure 6 shows that when the impedance between the lead and the case is changed, the resonance peak of the dissipated power shifts to a different length, and the peak value at the resonance peak decreases. However, one should note the second resonance peak in these curves. As the value of the first resonance peak decreases, the value of the second resonance peak increases. 


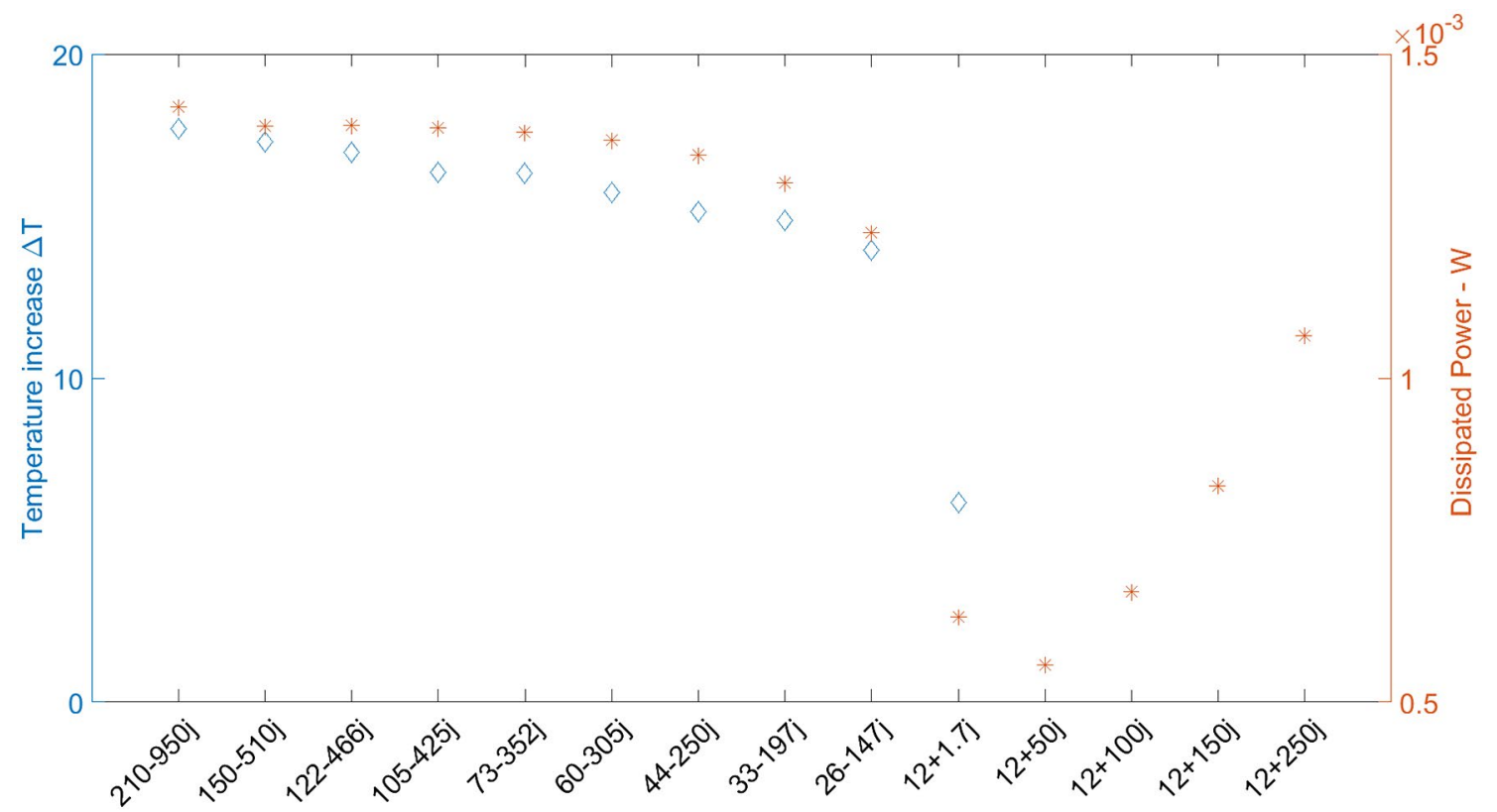

F I G URE 5 The calculated dissipated power for the implemented impedance values is plotted as red stars. The temperature rise for these impedance values, which are measured in the experiments, are plotted as blue diamonds

F I G URE 6 The imaginary part of the lead-case impedance is swept from $-j 50 \Omega$ to $j 50 \Omega$, and the dissipated power is calculated with respect to lead length. The line composed of light blue stars corresponds to setting $Z_{c}$ to 0 . The arrow shows the direction of the change in the power-length curve as $Z_{c}$ increases in $j 10 \Omega$ steps

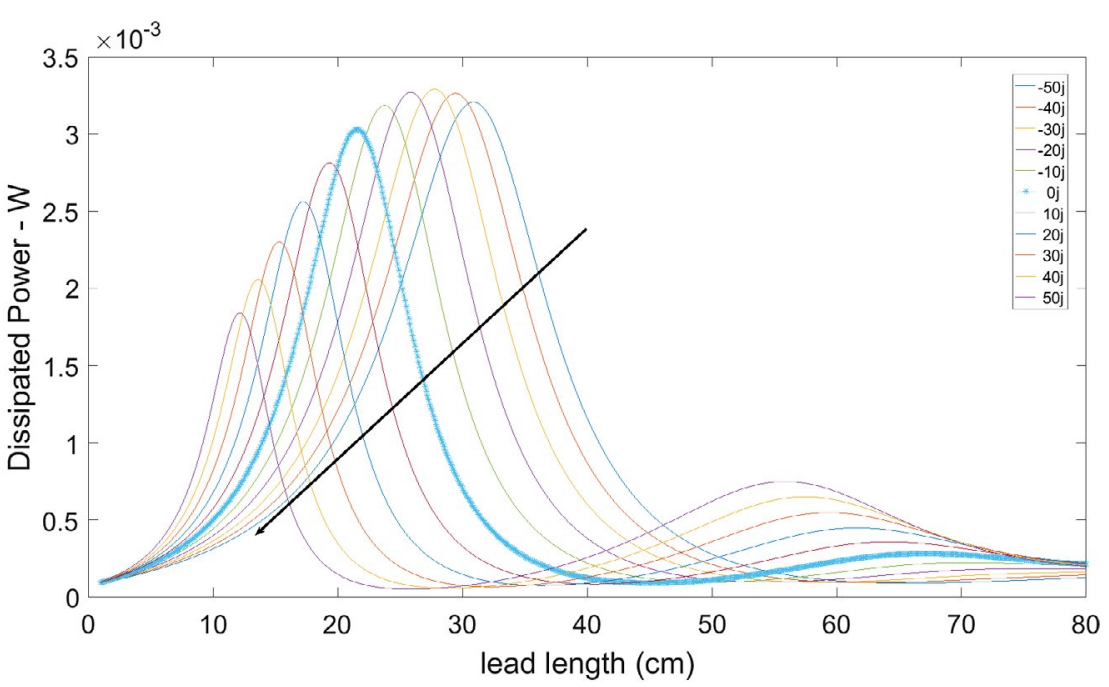

Another important point is that the properties of the surrounding tissue will affect the results of changing the value of the lead-case impedance. In Figure 7A,B, the value of the dissipated power with respect to the lead-case impedance is plotted for 40 and $90 \mathrm{~cm}$ leads, respectively, for media with conductivity values ranging from 0.2 to $0.7 \mathrm{~S} / \mathrm{m}$, while the relative permittivity was kept as 80 . Although the results behave similarly, the amount of change in the power versus impedance differs. Additionally, it is shown that the temperature rise for leads with different lengths will be affected differently for the same impedance changes (Figures 4, 6, and 7). A similar analysis was also carried out for media with relative permittivity values ranging from 50 to $90(\sigma=0.2 \mathrm{~S} / \mathrm{m})$ with the results presented in Figure 7C,D. Similarly, the temperature rise is affected by the initial lead length and the tissue permittivity to the lead-case impedance value.

The real-time wireless lead-case impedance control experimental results are given in Figure 8. The lead-case impedance value was at the lowest value, $12+j 1.7 \Omega$, at the start of the experiment. However, it was changed to the highest lead-case impedance value, 210 - j950 $\Omega, 360$ seconds later. Immediately after, the temperature changed rapidly. After another 223 seconds, the lead-case impedance value was set to the lowest value again, and a rapid decrease in temperature was observed. Finally, the lead-case impedance value was set to $26-j 147 \Omega$ for 265 seconds. The temperature changed again, but it did not rise to the value of the highest impedance value, although the RF exposure time was greater. 


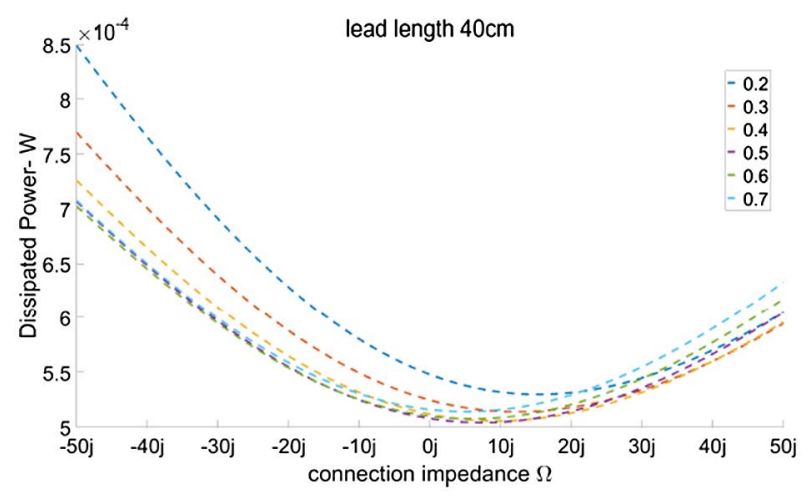

(A) Lead length $40 \mathrm{~cm}$.

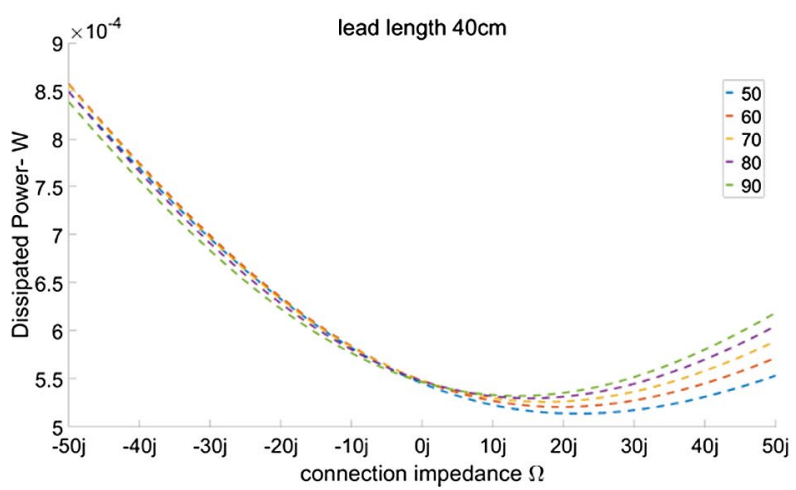

(C) Lead length $40 \mathrm{~cm}$.

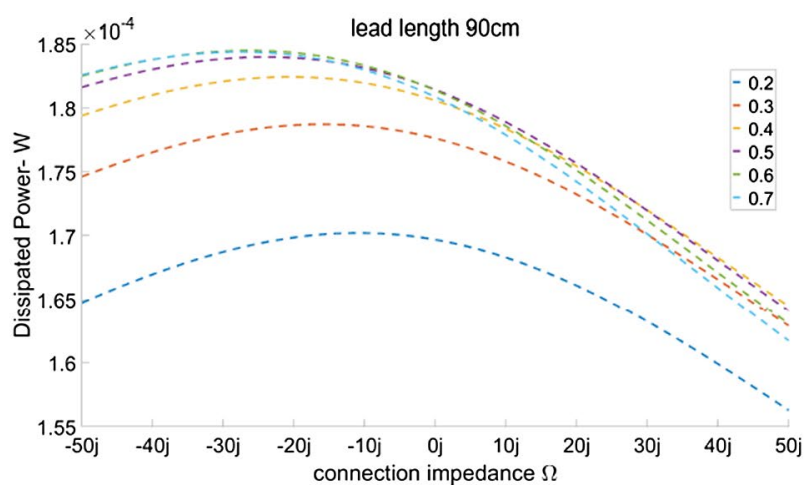

(B) Lead length $90 \mathrm{~cm}$.

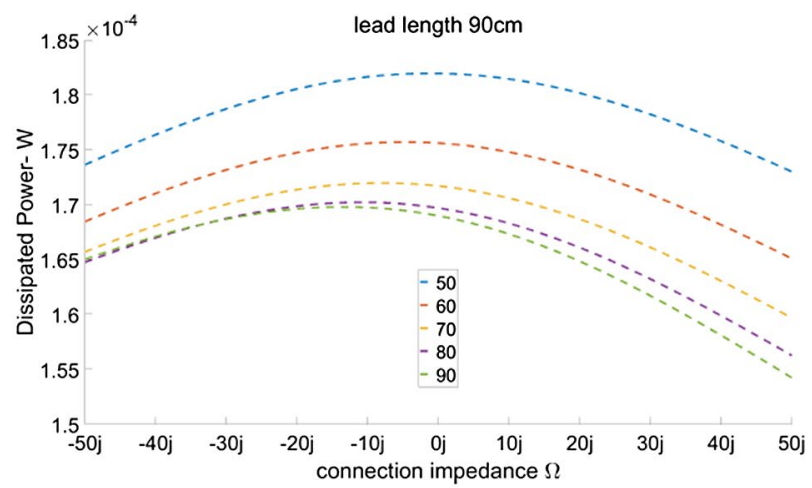

(D) Lead length $90 \mathrm{~cm}$.

F I G U RE 7 Effect of the lead-case impedance on the dissipated power. The imaginary part of the lead-case impedance $\left(Z_{c}+Z_{L C}\right)$ is swept from $-j 100 \Omega$ to $j 100 \Omega$ while the real part was kept as $291 \Omega$. In (A) and (B), the conductivity of the tissue was swept between 0.2 and $0.7 \mathrm{~S} / \mathrm{m}$ in 0.1 increments, while the relative permittivity was kept as 80 for lead lengths 40 and $90 \mathrm{~cm}$, respectively. In (C) and (D), the relative permittivity of the tissue was swept between 50 and 80 in increments of 10 while the conductivity was kept as $0.2 \mathrm{~S} / \mathrm{m}$ for lead lengths 40 and $90 \mathrm{~cm}$

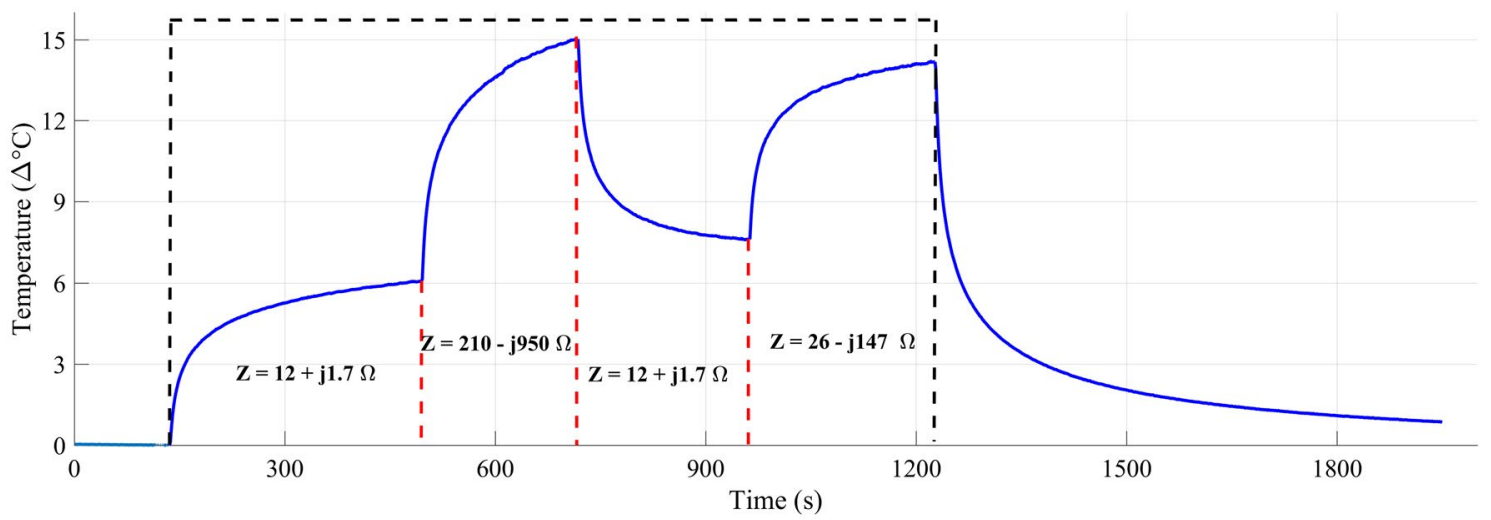

F I G U RE 8 The MR heating experiment illustrates the real-time wireless control of the RF properties of the AIMD during an experimental sequence. Note that the break points are indicated with red-dashed lines on the temperature curve where the lead-case impedance was switched to a different value. The corresponding impedance values are given in the area below the curve. RF started at 137 seconds and stopped at 1227 seconds, indicated with the black-dashed line. Note that all MR parameters are the same during the sequence

\section{5 | DISCUSSION AND CONCLUSION}

In this article, the effect of the impedance between the lead and the case of an AIMD is analyzed, and a new method that adjusts this parameter to reduce the risk of MRI for patients with these implants is presented. It is shown that by changing the lead-case impedance, electrode tip heating can be manipulated. However, the lead-case impedance that is required for minimum heating depends on many factors, 
including the position of the lead. Because we do not have access to lead-case impedance data for commercial devices in Figure 7, lead-case impedance was swept from -j50 to $j 50$ to show that their effect would be different for different lead lengths. Therefore, determining the lead-case impedance that minimizes electrode heating cannot be determined before implanting the AIMDs in the patients. To overcome this problem, we suggested a variable lead-case impedance to control the heating. As a proof of concept, we used 10 different leadcase impedance values. For the special cases that are tested in this article, these 10 different lead-case impedance values were enough to control the electrode tip temperature.

It is likely that the required lead-case impedance values depend on the geometry of the AIMD case, leads and electrodes. In this study, only capacitors are used, but it is possible that for some cases, inductors may be needed. It is also important to observe from Figure 3 that the temperature rise may change significantly for a small change in the leadcase impedance. Interestingly, around the minimum heating condition (see Figure 6), small variations in the lead-case impedance do not cause significant changes in the heating. Therefore, only using an on/off switch between the case and lead may be sufficient; however, we do not have enough data to determine this for all cases. As shown in Figure 7A,C, dissipated power started to increase at a certain inductive lead-case connection. Also in Figure 5, dissipated power calculations were carried on for inductive lead-case impedances. As calculations showed, further increasing the inductance might decrease the temperature rise, but after a certain value it would start to increase again. Therefore, the value of the lead-case connection is also critical. Determining the actual range and number of lead-case impedance values that provide safe scanning requires an extensive study that is beyond the scope of this article. Detecting coil-implant coupling by impedance measurement of RF coil has been investigated ${ }^{38}$ previously, which may be used for developing a method to determine the required impedance values outside of an MRI scanner. However, it requires extensive analysis, so it would be a focus for future studies.

It should be noted that in our design, the RF switches played an essential role in the lead-case impedance. While measuring the lead-case impedance, we accounted for the losses and parasitic capacitance values introduced by these switches. Note that when selecting the RF switches, in addition to the insertion loss of the switches and the parasitic capacitance that they introduce, their size, power consumption, and power handling capacity are important. It should be noted that the RF switches are the sole active components that are different than a previously designed implant, which has been evaluated during in vitro and in vivo MRI. ${ }^{32}$ Although the upgraded implant has been used without electrical problems while the gradient and RF system was operating together, the RF-only sequence that was used as the focus of the article was to evaluate RF induced tissue heating.

Additionally, it is important to emphasize that the proposed method is not a current-nulling method. As can be noted from the MRI heating experiments and corresponding MoTLiM simulations (Figure 5), the temperature is decreased by $65 \%$, but is not cancelled completely. Another limitation is that the method may be ineffective for electrically long leads. Because the reflected current waves from the lead-case connection will decay before reaching the electrode end on electrically long leads, it may have no or minimal effect on tip temperature. Hence, for higher field strengths, this method may be less efficient.

During the experiments, the states of the switches were manually changed by the experimenter while monitoring real-time temperature data. It should be noted that an automated program can be developed and run on a controller, or even by the MRI scanner itself. If a link between the MRI scanner and the implant is established, other methods, such as analysis of the image artifact around the lead, ${ }^{32,39,40}$ could be used to control the lead-case impedance; however, the image artifact signal would be an indirect measurement. Hence, the considerations discussed in the article ${ }^{40}$ should be addressed carefully for that method.

Other MR safety issues, such as the RF heating of the active MR catheters, may benefit from this technique because their usage is also limited because of RF-induced heating in the wire during MR interventions. In a recent study, Ozen et al investigated the idea by solving the inhomogeneous wave equations using Green's function and compared the results using transfer function measurements. ${ }^{41}$ They found $95 \%$ SAR variation for the worst-case and the best-case scenarios, depicting a similar approach that can be useful for active catheter safety.

A method for improving the RF safety of AIMDs by controlling the lead-case impedance was presented in this article. The effectiveness of the method was tested using simulations and experiments. A prototype AIMD with a wireless connection and embedded tip temperature sensor was used in the experiments. Using this method, it is shown that the induced RF currents in the leads of the AIMD can be altered in real time, which may reduce the RF safety concerns of patients with AIMDs during an MRI scan.

\section{ACKNOWLEDGMENTS}

The authors thank Ugur Yilmaz for help with RF sequence preparation.

\section{ORCID}

Volkan Acikel (D) https://orcid.org/0000-0003-2391-4545

Berk Silemek (D) https://orcid.org/0000-0001-8227-3632 


\section{REFERENCES}

1. Henderson JM, Tkach J, Phillips M, Baker K, Shellock FG, Rezai AR. Permanent neurological deficit related to magnetic resonance imaging in a patient with implanted deep brain stimulation electrodes for Parkinson's disease: case report. Neurosurgery. 2005;57:E1063; discussion, E1063.

2. Spiegel J, Fuss G, Backens M, et al. Transient dystonia following magnetic resonance imaging in a patient with deep brain stimulation electrodes for the treatment of Parkinson disease. J Neurosurg. 2003;99:772-774.

3. Achenbach S, Moshage W, Diem B, Bieberlea T, Schibgilla V, Bachmann K. Effects of magnetic resonance imaging on cardiac pacemakers and electrodes. Am Heart J. 1997;134:467-473.

4. Nyenhuis JA, Park S-M, Kamondetdacha R, Amjad A, Shellock FG, Rezai AR. MRI and implanted medical devices: basic interactions with an emphasis on heating. IEEE Trans Device Mater Reliab. 2005;9(5):467-480.

5. Yeung CJ, Susil RC, Atalar E. RF heating due to conductive wires during MRI depends on the phase distribution of the transmit field. Magn Reson Med. 2002;48:1096-1098.

6. Yeung CJ, Susil RC, Atalar E. RF safety of wires in interventional MRI: using a safety index. Magn Reson Med. 2002;47:187-193.

7. Yeung CJ, Atalar E. A Green's function approach to local rf heating in interventional MRI. Med Phys. 2001;28:826-832.

8. Park SM, Kamondetdacha R, Nyenhuis JA. Calculation of MRIinduced heating of an implanted medical lead wire with an electric field transfer function. J Magn Reson Imaging. 2007;26: 1278-1285.

9. Liu J, Zheng J, Wang Q, Kainz W, Chen J. A transmission line model for the evaluation of MRI RF-induced fields on active implantable medical devices. IEEE Trans Microw Theory Techn. 2018;66:4271-4281.

10. Feng S, Qiang R, Kainz W, Chen J. A Technique to Evaluate MRIinduced Electric Fields at the Ends of Practical Implanted Lead. IEEE Trans Microw Theory Techn. 2015;63:305-313.

11. Tokaya JP, Raaijmakers AJE, Luijten PR, Bakker J, Berg CAT. MRI-based transfer function determination for the assessment of implant safety. Magn Reson Med. 2017;78:2449-2459.

12. Tokaya JP, Raaijmakers AJE, Luijten PR, Berg CAT. MRIbased, wireless determination of the transfer function of a linear implant: Introduction of the transfer matrix. Magn Reson Med. 2018;80:2771-2784.

13. Acikel V, Atalar E. Modeling of radio-frequency induced currents on lead wires during MR imaging using a modified transmission line method. Med Phys. 2011;38:6623-6632.

14. Ladd ME, Quick HH. Reduction of resonant RF heating in intravascular catheters using coaxial chokes. Magn Reson Med. 2000;43:615-619.

15. Greatbatch W, Miller V, Shellock FG. Magnetic resonance safety testing of a newly-developed fiber-optic cardiac pacing lead. J Magn Reson Imaging. 2002;16:97-103.

16. Gray RW, Bibens WT, Shellock FG. Simple design changes to wires to substantially reduce MRI-induced heating at $1.5 \mathrm{~T}$ : implications for implanted leads. Magn Reson Imaging. 2005;23:887-891.

17. Bottomley PA, Kumar A, Edelstein WA, Allen JM, Karmarkar PV. Designing passive MRI-safe implantable conducting leads with electrodes. Med Phys. 2010;37:3828-3843.

18. Elwassif MM, Datta A, Rahman A, Bikson M. Temperature control at DBS electrodes using a heat sink: experimentally validated FEM model of DBS lead architecture. J Neural Eng. 2012;9:046009.
19. Serano P, Angelone LM, Katnani H, Eskandar E, Bonmassar G. A novel brain stimulation technology provides compatibility with MRI. Sci Rep. 2015;5:9805.

20. Bottomley PA, EdelsteinWA KA, Allen JM, Karmarkar P. Resistance and inductance based MRI-safe implantable lead strategies. In Proceedings of the 17th Annual Meeting of the ISMRM, Honolulu, HI, 2009. p. 4805.

21. Sutton R, Kanal E, Wilkoff BL, et al. Safety of magnetic resonance imaging of patients with a new Medtronic EnRhythm MRI SureScan pacing system: clinical study design. Trials. 2008;9:68.

22. Mitka M. First MRI-safe pacemaker receives conditional approval from FDA. JAMA. 2011;305:985-986.

23. Wilkoff BL, Bello D, Taborsky M, et al. Magnetic resonance imaging in patients with a pacemaker system designed for the magnetic resonance environment. Heart Rhythm. 2011;8:65-73.

24. Eryaman Y, Akin B, Atalar E. Reduction of implant RF heating through modification of transmit coil electric field. Magn Reson Med. 2011;65:1305-1313.

25. Golestanirad L, Iacono MI, Keil B, et al. Construction and modeling of a reconfigurable MRI coil for lowering SAR in patients with deep brain stimulation implants. NeuroImage. 2017;147:577-588.

26. Golestanirad L, Keil B, Angelone LM, Bonmassar G, Mareyam A, Wald LL. Feasibility of using linearly polarized rotating birdcage transmitters and close-fitting receive arrays in MRI to reduce SAR in the vicinity of deep brain simulation implants. Magn Reson Med. 2017;77:1701-1712.

27. Eryaman Y, Guerin B, Akgun C, et al. Parallel transmit pulse design for patients with deep brain stimulation implants. Magn Reson Med. 2015;73:1896-1903.

28. Gudino N, Sonmez M, Yao Z, et al. Parallel transmit excitation at $1.5 \mathrm{~T}$ based on the minimization of a driving function for device heating. Med Phys. 2014;42:359-371.

29. Etezadi-Amoli M, Stang P, Kerr A, Pauly J, Scott G. Controlling radiofrequency-induced currents in guidewires using parallel transmit. Magn Reson Med. 2015;74:1790-1802.

30. McElcheran CE, Yang B, Anderson KJT, Golenstani-Rad L, Graham SJ. Investigation of parallel radiofrequency transmission for the reduction of heating in long conductive leads in 3 Tesla magnetic resonance 1maging. PLOS ONE. 2015;8(10):e0134379.

31. Eryaman Y, Kobayashi N, Moen S, et al. A simple geometric analysis method for measuring and mitigating RF induced currents on deep brain stimulation leads by multichannel transmission/ reception. NeuroImage. 2019;184:658-668.

32. Silemek B, Acikel V, Oto C, et al. A temperature sensor implant for active implantable medical devices for in vivo subacute heating tests under MRI. Magn Reson Med. 2018;79:2824-2832.

33. Acikel V, Uslubas A, Atalar E. Modeling of electrodes and implantable pulse generator cases for the analysis of implant tip heating under MR imaging. Med Phys. 2015;42:3922-3931.

34. Acikel V. Analysis of current induction on thin conductors inside the body during MRI scan. Ankara, Turkey: Bilkent University; 2014.

35. Wong KL. Planar antennas for wireless communications. Hoboken, NJ: Wiley-Interscience; 2003:301.

36. Gao V. Proximity And RSSI I Bluetooth Technology Website. 2015; https://blog.bluetooth.com/proximity-and-rssi. Accessed June 20, 2019.

37. Nordbeck P, Fidler F, Weiss I, et al. Spatial distribution of RFinduced E-fields and implant heating in MRI. Magn Reson Med. 2008;60:312-319. 
38. Ellenor CW, Stang PP, Etezadi-Amoli M, Pauly JM, Scott GC. Offline impedance measurements for detection and mitigation of dangerous implant interactions: an RF safety prescreen. Magn Reson Med. 2015;73:1328-1339.

39. van den Bosch MR, Moerland MA, Lagendijk JJW, Bartels LW, van den Berg CAT. New method to monitor RF safety in MRIguided interventions based on RF induced image artefacts. Med Phys. 2010;37:814-821.

40. Eryaman Y, Turk EA, Oto C, Algin O, Atalar E. Reduction of the radiofrequency heating of metallic devices using a dual-drive birdcage coil. Magn Reson Med. 2013;69:845-852.
41. Özen AC, Lottner T, Bock M. Safety of active catheters in MRI: termination impedance versus RF-induced heating. Magn Reson Med. 2019;81:1412-1423.

How to cite this article: Acikel V, Silemek B, Atalar E. Wireless control of induced radiofrequency currents in active implantable medical devices during MRI. Magn Reson Med. 2020;83:2370-2381. https://doi.org/10.1002/mrm.28089 